\section{Egatso} Thoracic Surgery, Catholic University, Rome, Italy

\section{Correspondence to} Dr Marco Chiappetta Department of General Thoracic Surgery, Catholic University, Rome 00168, Italy; marco_chiappetta@yahoo.it, marcokiaps@hotmail.it

Received 23 November 2015 Revised 19 January 2016 Accepted 20 January 2016 Published Online First 12 February 2016

\title{
Multiple giant bullae of the lung mimicking massive pneumothorax in a patient with osteogenesis imperfecta
}

\author{
Marco Chiappetta, Dania Nachira, Venanzio Porziella, Maria Letizia Vita, \\ Stefano Margaritora
}

A 60-year-old Caucasian man was admitted to our emergency department with progressive dyspnoea, chronic cough and general asthenia. Physical examination revealed lack of left chest wall motion and absent breath sounds over the left hemithorax.

Vital signs were stable with a heart rate of $90 \mathrm{bpm}$, blood pressure of $120 / 70 \mathrm{~mm} \mathrm{Hg}$, respiratory rate of 20 breaths per minute and oxygen saturation by pulse oximetry of $95 \%$ while breathing room air.

The patient reported being an active smoker with a 40 pack-year smoking history. $\mathrm{He}$ also had a history of type IV osteogenesis imperfecta, a connective tissue pathology that could be associated with lung abnormalities. ${ }^{12}$

A chest radiograph (figure 1A) was suggestive of a left massive pneumothorax that may require emergency insertion of a chest tube thoracostomy. However, a distinct pleural line could not be

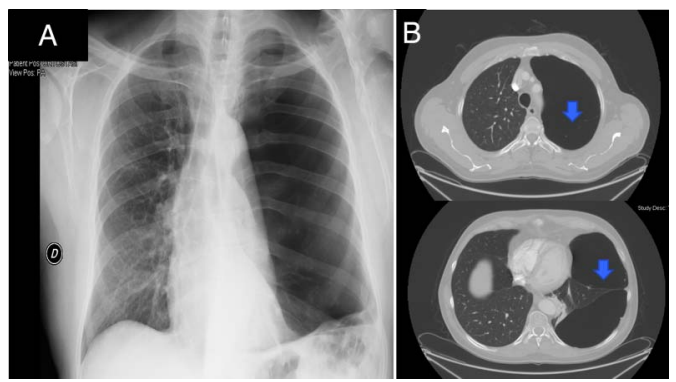

Figure 1 Chest radiograph suggestive of a left massive pneumothorax (A) and CT scan revealing two giant bullae with internal division and septae separated by a small area of lung parenchyma (B). identified on the chest radiograph and bronchovascular pattern was visible in the left base of the lung.

Therefore, the patient underwent a CT scan, which revealed two giant bullae of the left upper and lower lobes with multiple intrabullae septae (figure 1B). Though chest ultrasonography could not be performed due to lack of equipment in the emergency department, it could have proved useful to identify pleural sliding. However, given that the patient was haemodynamically stable and not in significant respiratory distress, we opted to obtain the definitive diagnostic test, which is a chest CT scan. ${ }^{3}$

The patient underwent left thoracotomy for the resection of the two giant bullae (figure $2 \mathrm{~A}, \mathrm{~B}$ ). This led to a complete re-expansion of the left lung after surgery (figure 2C). The patient was discharged after 5 days without any postoperative complications. Of note, the $\alpha 1$-antitripsin level was normal.

In conclusion, we report an interesting and previously unreported case of osteogenesis imperfecta and giant bullae successfully treated by bullae resection.

Competing interests None declared.

Provenance and peer review Not commissioned; externally pee reviewed.

\section{REFERENCES}

1 Shapiro JR, Burn VE, Chipman SD, et al. Pulmonary hypoplasia and osteogenesis imperfecta type II with defective synthesis of alpha I (1) procollagen. Bone 1989;10:165-71.

2 Morikawa M, Fukuda Y, Terasaki Y, et al. Osteogenesis imperfecta associated with dendriform pulmonary ossification. Am J Respir Crit Care Med 2016;193:460-1.

3 Gelabert C, Nelson M. Bleb point: mimicker of pneumothorax in bullous lung disease. West J Emerg Med 2015;16:447-9.

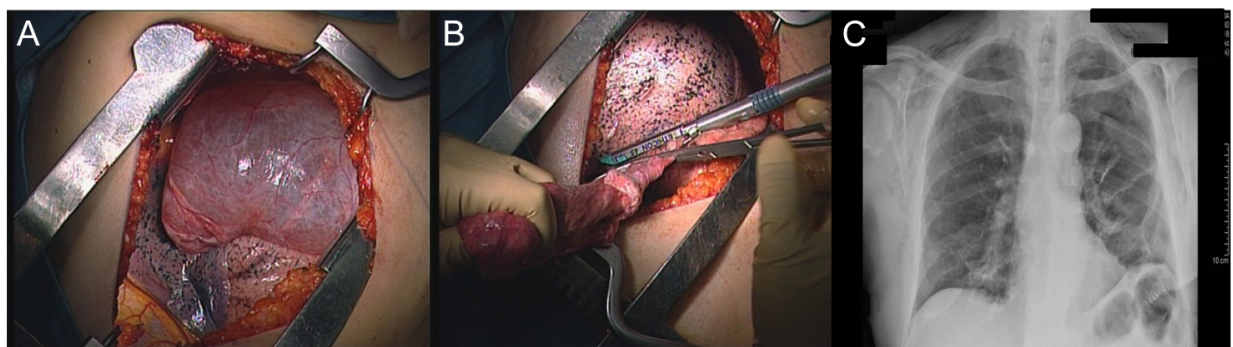

Figure 2 View of the giant bullae during surgery when it is inflated $(A)$ and when air flow is interrupted before resection with the whole lung re-expanded (B). The postoperative chest radiograph shows left lung expansion (C). 